\title{
PTEN inhibits the migration and invasion of HepG2 cells by coordinately decreasing MMP expression via the PI3K/Akt pathway
}

\author{
TAO TIAN, KE-JUN NAN, HUI GUO, WEN-JUAN WANG, ZHI-PING RUAN, \\ SHU-HONG WANG, XUAN LIANG and CHUANG-XIN LU \\ Department of Oncology, First Affiliated Hospital of Medical College of \\ Xi'an Jiaotong University, Xi'an 710061, Shaanxi Province, P.R. China
}

Received January 25, 2010; Accepted March 8, 2010

DOI: $10.3892 /$ or_00000800

\begin{abstract}
Hepatocellular carcinoma (HCC) is one of the most common malignant tumors. Most patients with HCC die within one year after diagnosis largely because of frequent tumor recurrence and metastasis. The tumor suppressor gene PTEN (phosphatase and tensin homolog deleted on chromosome 10) is one of the most commonly lost or mutated genes in a variety of human cancers, including HCC. PTEN antagonizes phosphoinositide-3-kinase (PI3K)/ATP-dependent tyrosine kinase (Akt) signaling, thereby negatively regulating a multitude of biological aggressive tumor behaviors. However, the direct role and mechanism of PTEN in the regulation of invasion and invasion-related gene expression in HCC remain to be elucidated. In this study, we introduced wild-type PTEN or phosphatase-dead PTEN into HepG2 cells that have low expression of PTEN. We found that overexpression of PTEN inhibits HepG2 cell growth via cell cycle arrest without inducing apoptosis. Matrigel invasion and scratch assays indicated that PTEN significantly inhibits HepG2 cell migration and invasion in vitro. On the molecular level, overexpression of PTEN suppressed expression of matrix metalloproteinase (MMP)-2 and -9 in HepG2 cells. Similarly, treatment of HepG2 cells with the PI3K/Akt pharmacological inhibitor, LY294002, potently suppressed cell migration and invasion as well as expression of MMPs. However, the phosphatase-dead PTEN mutant did not exert the same effects. Our data show that PTEN not only inhibits HepG2 cell growth via cell cycle arrest, but also suppresses cell invasion in a PI3K/Akt/MMP-dependent manner, which suggests that loss or mutation of PTEN may contribute to increased cell invasion and facilitates HCC progression.
\end{abstract}

Correspondence to: Dr Ke-Jun Nan, Department of Oncology, First Affiliated Hospital of Medical College of Xi'an Jiaotong University, Xi'an 710061, Shaanxi Province, P.R. China

E-mail: nankj@163.com

Key words: hepatocellular carcinoma, PTEN, cell invasion, cell growth, matrix metalloproteinase

\section{Introduction}

Hepatocellular carcinoma (HCC) is one of the most frequently diagnosed malignant tumors and is responsible for $>600,000$ deaths annually (1). Despite improvements in treatment modalities during the past few decades, most patients with HCC die within one year of diagnosis, largely because of the aggressive biological characteristics of the tumors, including rapid tumor growth, frequent tumor recurrence and metastasis. Clearly, there is an urgent need for further insights into the molecular mechanisms responsible for the aggressive biological behavior of HCC.

Tumor invasion and metastasis are complex, multistage processes by which cancer cells undergo genetic alternations that result in the acquisition of their ability to degrade and migrate through the extracellular matrix $(\mathrm{ECM})(2,3)$. Matrix metalloproteinases (MMPs) are a family of zinc-dependent endopeptidases that are capable of degrading all essential components of the ECM, including type IV collagen, laminin, entactin, proteoglycans and glycosaminoglycans $(4,5)$. MMP-2 and MMP-9 are particularly effective at degrading the basement membrane and have been previously shown to facilitate tumor invasion and metastasis (4,6-9). Recent reports from clinical pathological studies have demonstrated that upregulation of MMP-2 and MMP-9 mRNA levels correlates with later TNM staging and metastasis in HCC (10) and their expression may be helpful in predicting poor prognosis in HCC patients after live transplantation (11). In addition, recent anti-metastasis experiments, such as NF-кB inhibition (12), treatment with pravastatin (13) and Spred-1 overexpression (14), have suggested that the invasive and metastatic abilities of HCC are suppressed by decreased expression of MMP-2 and MMP-9. These results suggest that MMP-2 and MMP-9 play an important role in HCC invasion and metastasis. However, the mechanism by which MMP expression is controlled in HCC remains unclear.

PTEN (phosphatase and tensin homolog deleted on chromosome 10) has been described as a tumor suppressor gene due to its frequent loss or mutation in a variety of human cancers, including HCC $(15,16)$. In mice, liver-specific loss of PTEN leads to the development of HCC by 74 weeks of age (17). In human patients, the degree of PTEN expression in HCC is linked to tumor differentiation, invasion, metastasis 
and patient survival (18), indicating that PTEN has potentially suppressive effects on HCC progression (19). Growing evidence suggests that PTEN regulates a multitude of biological aggressive tumor behaviors, such as growth, selfrenewal and metastasis (20-22). Specifically, it has been proposed that PTEN regulates these tumor behaviors through its lipid phosphatase activity, which mediates the dephosphorylation of phosphatidylinositol 3,4,5-trisphosphate and its conversion to phosphatidylinositol-4,5-bisphosphate, thereby opposing the activity of phosphoinositide-3-kinase (PI3K)/ATP-dependent tyrosine kinase (Akt). Activation of the PI3K/Akt pathway may potentiate cell survival, proliferation, apoptosis and migration $(23,24)$.

In $\mathrm{HCC}$, deregulation of this signaling pathway has been clearly shown to promote cell cycle progression and cancer formation (19). Several recent studies have shown that activation of the PI3K/Akt signaling pathway correlates with increased expression of MMP-2 and MMP-9 and promotes cancer cell invasion (25). In addition, it has been shown that inhibition of Akt activation by using shRNA or a pharmacological inhibitor significantly decreases the invasive ability of cancer cells by downregulating the expression of MMP-2 and MMP-9 $(26,27)$. Hence, we hypothesize that activation of PI3K/Akt signaling, in the absence of PTEN, can increase HCC cell migration and invasion and the expression of MMPs.

In the present study, we introduced wild-type PTEN or phosphatase-dead PTEN mutant into PTEN-deficient HepG2 cells and used a PI3K/Akt pharmacological inhibitor, LY294002, to test our hypothesis. In addition, to understand the molecular mechanisms underlying the suppressive effects of PTEN, we examined the levels of MMP-2 and MMP-9 mRNA and protein expression. Furthermore, we analyzed the levels of Akt phosphorylation with respect to the invasive activity of HepG2 cells.

\section{Materials and methods}

Cell culture and transfection. HCC cell lines HepG2, Bel7402 and SMMC-7721 were cultured in RPMI-1640 medium supplemented with $10 \%$ fetal bovine serum (FBS) in $5 \% \mathrm{CO}_{2}$ at $37^{\circ} \mathrm{C}$. The $\mathrm{HCC}$ cell line MHCC97 and the normal hepatic cell line L02 were cultured in a humidified $5 \% \mathrm{CO}_{2} / 95 \%$ air atmosphere in DMEM medium supplemented with 10\% FBS.

The wild-type PTEN plasmid (PTEN) and phosphotasedead PTEN mutant plasmid (PTEN.C124S), which harbors a cysteine to serine mutation at position 124 , as previously described (28), were gifts from Professor Yuxin Yin (Columbia University, NY, USA). Stable transfection of the cells with PTEN, PTEN.C124S, or pcDNA3.1 empty plasmid (Invitrogen, CA, USA) was performed with Lipofectamine ${ }^{\mathrm{TM}}$ 2000 (Invitrogen) reagent according to the manufacturer's guidelines. After transfection for $24 \mathrm{~h}$, cells were diluted 1:20 and selected in media supplemented with $500 \mu \mathrm{g} / \mathrm{ml}$ of hygromycin (Sigma, MO, USA) for 1-2 months. Stable transfectants were confirmed by Western blotting and maintained in media supplemented with $250 \mu \mathrm{g} / \mathrm{ml}$ hygromycin.

Cell proliferation assay. Cells were seeded into 96-well plates (5,000 cells/well). 3-(4,5-Dimethylthiazol-2-yl)-2,5- diphenyltetrazolium bromide (MTT, Sigma) were added at 1 , $2,3,4,5,6,7$ days after seeding. After $4 \mathrm{~h}$ of incubation at $37^{\circ} \mathrm{C}, 150 \mu \mathrm{l}$ dimethyl sulfoxide (DMSO, Sigma) were added into each well and the absorbance was measured at $492 \mathrm{~nm}$ on a Multifunction microplate reader (PolarStar Optima BMG, Germany).

Cell cycle assay. Cells were harvested, fixed in ice-cold $70 \%$ ethanol for $30 \mathrm{~min}$ and stained with propidium iodide (PI, Sigma) for $30 \mathrm{~min}$ at room temperature. Cell cycle progression was assessed by flow cytometry (FACSCalibur BD, USA).

Cell apoptosis assay. Cells were harvested and resuspended in binding buffer. After addition of annexin V-FITC and PI (Jingmei Biotech Co., Ltd., Shenzhen, China), the cells were incubated for $30 \mathrm{~min}$ at room temperature and then analyzed by flow cytometry (FACSCalibur). Apoptotic cells were defined as being annexin $\mathrm{V}^{+} / \mathrm{PI}^{-}$.

In vitro scratch assay. Cell migration was assessed by using an 'in vitro scratch assay'. Briefly, cells were cultured in 6-well plates and grown into a monolayer. A pipette was used to scrape the cell monolayer in a straight line in order to create a scratch. In addition, the medium was replaced. For transfection experiments, transfected cells were cultured in fresh RPMI-1640 medium supplemented with 10\% FBS. For pharmacological inhibition assays, HepG2 cells were cultured in RPMI-1640 medium supplemented with 10\% FBS and either the PI3K/Akt inhibitor LY294002 (10 or $20 \mu \mathrm{M}$, Sigma) or DMSO (control). At initial time points $(0 \mathrm{~h})$ and at $24 \mathrm{~h}$ time points, scratches were observed using an inverted microscope (AE31, Motic, China) and the distance from one side of the scratch to the other was measured at different intervals using Image Pro-Plus 6.0 software (Media Cybernetics, USA).

Matrigel invasion assay. The invasion assay was performed using a Millicell invasion chamber $(8-\mu \mathrm{m}$ pore size, Millipore, MA, USA). Cells were resuspended in RPMI-1640 medium supplemented with $1 \%$ FBS and seeded into the upper well coated with Matrigel (Sigma). Simultaneously, RPMI-1640 medium supplemented with $10 \%$ FBS was placed in the lower well of the chamber. For transfection experiments, 10,000 cells were seeded into each chamber. For pharmacological inhibition assays, 10,000 cells were seeded into each chamber in the presence (10 or $20 \mu \mathrm{M}$ LY294002) or absence (DMSO) of inhibitor. The cells were allowed to invade the Matrigel for $24 \mathrm{~h}$ at $37^{\circ} \mathrm{C}$. After removal of cells from the upper surface of the chambers, the membranes were stained with crystal violet and observed using an inverted microscope (AE31). The number of invading cells per field was assessed by counting 10 random fields at x200 magnification.

Western blotting. Whole cell lysates were prepared in cell lysis buffer (40 mmol/1 Tris- $\mathrm{HCl} \mathrm{pH} 7.4,10 \%$ glycerol, $50 \mathrm{mmol} / \mathrm{l}$ BGP, $5 \mathrm{mmol} / 1 \mathrm{EGTA}, 2 \mathrm{mmol} / \mathrm{l}$ EDTA, $0.35 \mathrm{mmol} / \mathrm{l}$ vanadate, $10 \mathrm{mmol} / 1 \mathrm{NaF}$, and $0.3 \%$ Triton X-100) supplemented with protease inhibitors (Roche, Penzberg, Germany). Equivalent amounts of protein were loaded onto SDS-PAGE gels and transferred onto Immobilon membranes (Millipore). Membranes were incubated with the following primary 
A

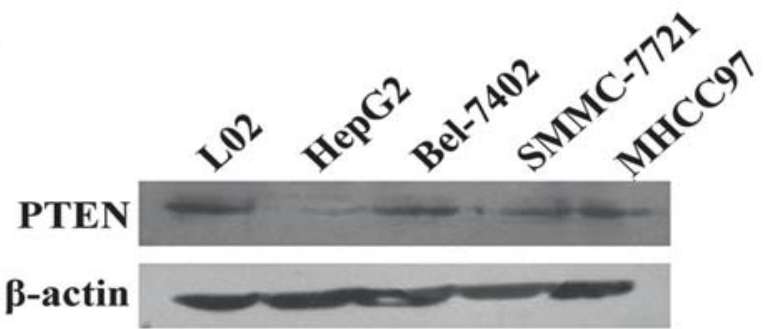

C
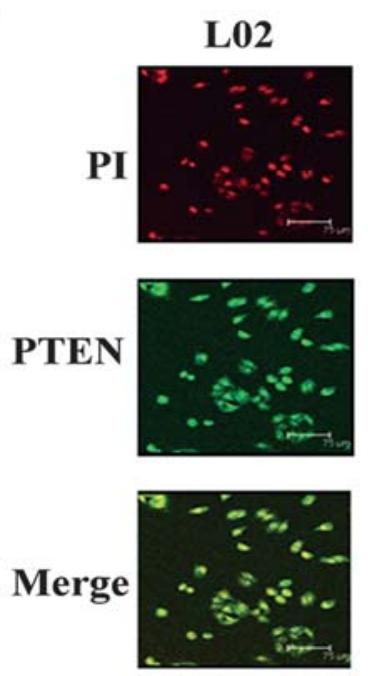
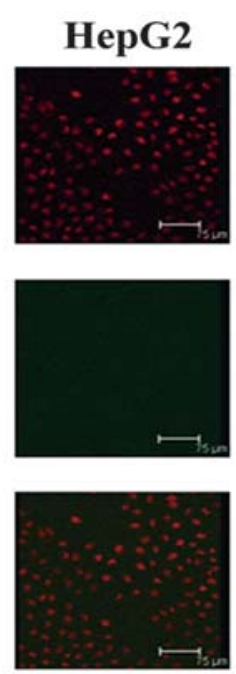
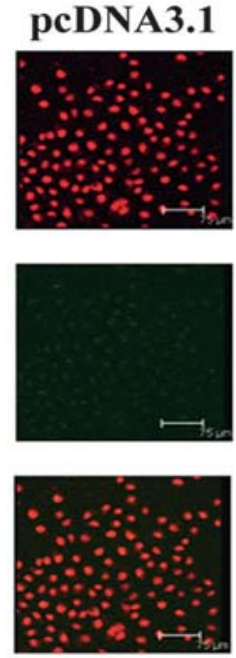

B
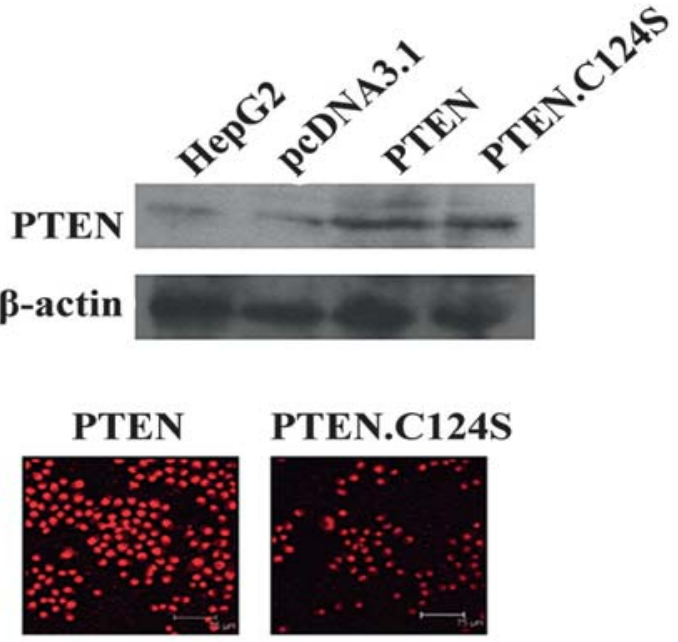

PTEN.C124S
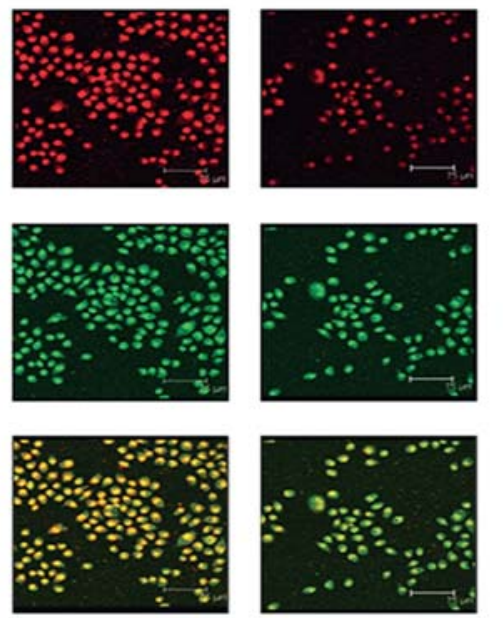

Figure 1. HepG2 cells stably express exogenous PTEN and its mutant. (A) Western blot analysis of PTEN expression in 4 hepatocellular carcinoma cell lines and 1 normal hepatic cell line. B-actin was used as an internal control. (B) Western blot analysis of PTEN expression after transfection of the cells with different plasmids. (C) Cells were fixed on coverslips and immunostained with anti-PTEN antibody, FITC-conjugated anti-mouse IgG (green) and PI (red). Scale bars: $75 \mu \mathrm{m}$.

antibodies: anti-PTEN (1:250), anti-Akt1/2 (1:500), antiMMP-2 (1:200), anti-MMP-9 $(1: 200)$ and anti- $\beta$-actin (1:800, all purchased from Santa Cruz Biotechnology, Santa Cruz, CA, USA) and anti-phospho-Akt (Ser473) (1:500; purchased from Cell Signaling, MA, USA). Proteins were visualised with horseradish peroxidase-conjugated secondary antibody (1:5,000; purchased from Santa Cruz Biotechnology) followed by ECL reaction (Millipore).

Quantification of MMP-2 and MMP-9. Cells were seeded at a density of $1 \times 10^{5}$ cells $/ \mathrm{ml}$ into 6 -well plates a day before the experiment. Transfected cells were cultured in fresh RPMI1640 medium supplemented with $1 \%$ FBS and parental HepG2 cells were cultured in fresh RPMI-1640 medium supplemented with 1\% FBS with or without LY294002 (10 or $20 \mu \mathrm{M})$. After $48 \mathrm{~h}$ of incubation, cell supernatants were collected and MMP-2 and MMP-9 concentrations were quantified using the ELISA kits (Shanghai Westang Bio-Tech Co., Ltd., Shanghai, China).

$R T$-PCR. Total mRNA was extracted using TRIzol reagent (Invitrogen). The cDNA synthesis was conducted using Takara RT-PCR kit (Dalian, China) according to the manufacturer's instructions. The PCR products were amplified with 35 cycles $\left(30 \mathrm{sec}\right.$ at $95^{\circ} \mathrm{C} ; 30 \mathrm{sec}$ at $60^{\circ} \mathrm{C} ; 1 \mathrm{~min}$ at $72^{\circ} \mathrm{C}$ ). The PCR primer sequences and the conditions are shown in Table I.
Immunofluorescence analysis. Cells were seeded onto glass slides at $1 \times 10^{5}$ cells $/ \mathrm{ml}$. The next day, cells were fixed with $4 \%$ paraformaldehyde for $30 \mathrm{~min}$, permeabilized with $0.1 \%$ Triton X-100 for $10 \mathrm{~min}$ and blocked with 5\% bovine serum albumin for $1 \mathrm{~h}$. The cells were then stained with anti-PTEN antibody $(1: 100)$ at $4^{\circ} \mathrm{C}$ overnight, followed by staining with FITC-conjugated anti-mouse immunoglobulin for $2 \mathrm{~h}$ and PI for $15 \mathrm{~min}$. Immunofluorescence was visualized with a laser scanning confocal microscope (TCS SP2, Leica, Germany).

Statistical analysis. All data are shown as the mean \pm standard deviation and analyzed using SPSS 13.0 software (SPSS Inc., IL, USA). Statistical significance was analyzed using the one-way ANOVA test. $\mathrm{P}<0.05$ was considered statistically significant.

\section{Results}

Expression and cellular localization of PTEN protein after transfection. To analyze PTEN expression in different HCC cells, we evaluated a panel of 4 human HCC lines (HepG2, Bel-7402, SMMC-7721 and MHCC97) and one normal hepatic cell line (L02 as positive control). As shown in Fig. 1A, the abundance of PTEN varied between the different cell lines, with HepG2 having relatively low levels of the protein, as previously reported $(29,30)$. Therefore, we used the HepG2 cell line to carry out subsequent experiments. The expression 
A

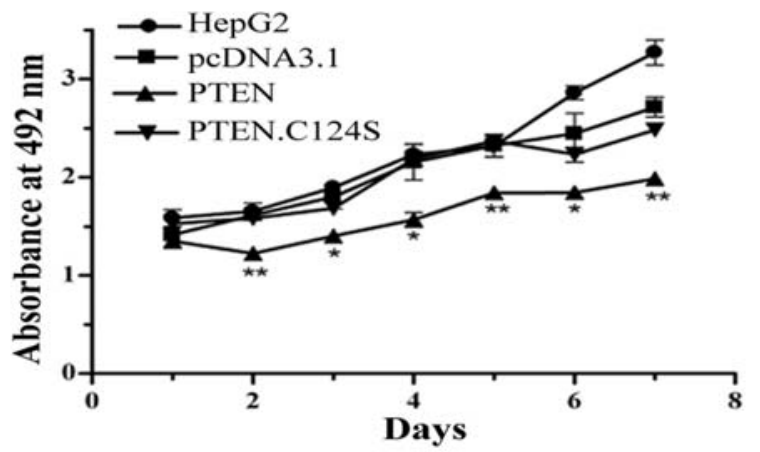

B
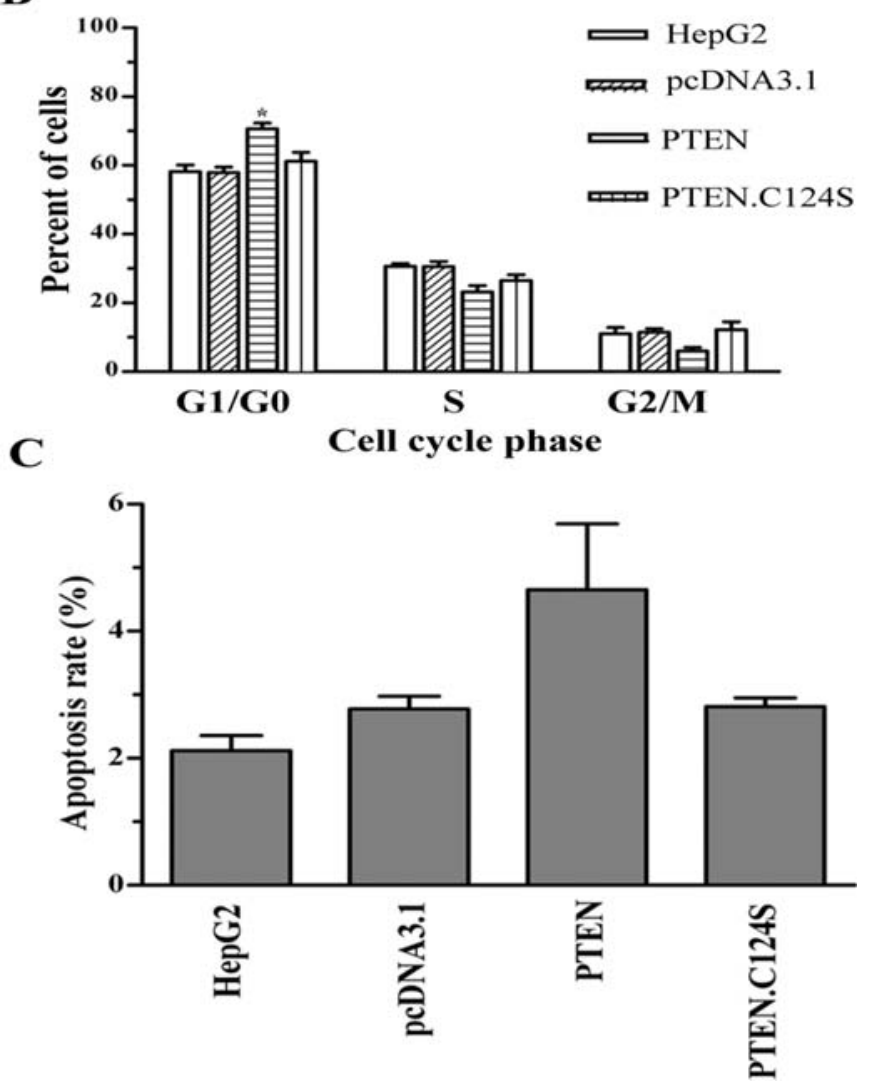

Figure 2. PTEN inhibits cell proliferation via G1 cell cycle arrest without inducing apoptosis. (A) Cells were seeded into 96-well plates in RPMI-1640 medium supplemented with $10 \%$ FBS and were cultured for different periods of time. Cells were quantified by performing an MTT assay at the indicated time points. Points, mean $(\mathrm{n}=3)$; bars, SD; ${ }^{*} \mathrm{P}<0.05 ;{ }^{* *} \mathrm{P}<0.01$ compared to pcDNA3.1 controls. (B) Cells were harvested after $48 \mathrm{~h}$ and stained with PI. Cell cycle distribution was assessed by flow cytometry. Columns, mean $(n=3)$; bars, $\mathrm{SD} ;{ }^{*} \mathrm{P}<0.05$ compared to pcDNA3.1 controls. (C) Cells were harvested after $48 \mathrm{~h}$ and were stained with annexin V and PI. Apoptotic cells were defined as being annexin $\mathrm{V}^{+} / \mathrm{PI}^{-}$and were assessed by flow cytometry. Columns, mean $(n=3)$; bars, SD.

and cellular localization of PTEN protein following transfection was confirmed by Western blot and immunofluorescence analysis, respectively. While cells transfected with wild-type PTEN produced significant levels of PTEN protein and suppressed Akt phosphorylation at Ser473, cells transfected with the phosphatase-dead PTEN.C124S mutant produced PTEN protein at similar levels but did not affect Akt phosphorylation (Figs. 1B and 4E). Immunofluorescence analysis revealed that, similar to normal hepatic L02 cells,
PTEN was predominantly expressed in the cytosol and nucleus of HepG2 cells transfected with either PTEN or PTEN.C124S (Fig. 1C). Therefore, we succeeded in obtaining cells stably expressing exogenous PTEN and its mutant, mimicking endogenous PTEN expression and cellular localization.

Overexpression of PTEN inhibits growth of HepG2 cells. MTT assay was performed to evaluate the effect of PTEN inhibition on cell growth. The results showed that HepG2 cells transfected with PTEN grew slower than those transfected with empty plasmid (Fig. 2A). After 7 days of culture, but not on the first days of culture, different growth rates were observed in HepG2 cells transfected with PTEN when compared to cells transfected with the other plasmid (Fig. 2A). In contrast, HepG2 cells expressing PTEN.C124S did not exhibit a decrease in growth rate within 7 days of culture. Upon analysis of cell cycle distribution by flow cytometry, we found that overexpression of wild-type PTEN markedly increased the number of cells in the G0/G1 phase and resulted in a G1phase cell cycle arrest (Fig. 2B). However, we did not find a difference in the number of cells in the $\mathrm{S}$ or $\mathrm{G} 2 / \mathrm{M}$ phase between the transfected cells.

Analysis of apoptosis by annexin V-FITC/PI staining revealed that only a small number of $\mathrm{HepG} 2$ cells expressing wild-type PTEN underwent apoptosis (Fig. 2C), indicating that, rather than inducing apoptosis, PTEN inhibits cell growth by blocking the progression of cells from the G1 to the $\mathrm{S}$ phase of the cell cycle. To determine whether PTENmediated growth inhibition was attributed to the antagonizing activity of Akt signaling, we used HepG2 cells expressing PTEN.C124S and performed similar MTT assays. As shown in Fig. 2, PTEN.C124S-expressing HepG2 cells did not exhibit growth inhibition, cell cycle arrest or apoptosis. These findings suggest that PTEN inhibits the growth of HepG2 cells via PI3K/Akt-mediated cell cycle arrest.

PTEN inhibits HepG2 cell migration and invasion through the PI3K/Akt pathway. To determine whether PTEN inhibits migration and invasion of HepG2 cells, we analyzed the ability of transfected cells to respond to 10\% FBS by performing a Matrigel invasion assay. The results demonstrate that PTEN virtually suppresses cell invasion through the Matrigel (Fig. 3A). The mean invasiveness values were $57.8 \pm 11.12$ cells/field and $129.6 \pm 19.47$ cells/field for PTEN- or empty plasmid-transfected HepG2 cells, respectively $(\mathrm{P}<0.01$, Fig. 3B). However, the invasiveness of HepG2 cells expressing PTEN.C124S was not significantly different ( $\mathrm{P}>0.05$, Fig. 3B) from cells transfected with empty plasmid.

The highly specific PI3K/Akt inhibitor LY294002 was used to assess the role of the PI3K/Akt pathway in invasion. As shown in Fig. 4E, PTEN and LY294002 inhibited Akt phosphorylation, but the levels of total Akt protein remained unchanged. We also found that LY294002 suppressed Akt phosphorylation in a concentration-dependent manner (Fig. 4E). Treatment of the cells with $10 \mu \mathrm{M}$ LY294002 completely abolished Akt phosphorylation to the same extent as observed with expression of PTEN only (Fig. 4E). PTEN inhibited the ability of HepG2 cells to invade the Matrigel by 55\% (Fig. 3B) and $10 \mu \mathrm{M}$ LY294002 inhibited invasion by $64 \%$ (Fig. 3D). In addition, treatment of HepG2 cells 
Table I. RT-PCR primers and conditions.

\begin{tabular}{llcr}
\hline Gene & Primers & $\begin{array}{c}\text { Annealing } \\
\text { temperature }\left({ }^{\circ} \mathrm{C}\right)\end{array}$ & $\begin{array}{c}\text { Product } \\
\text { size }(\mathrm{bp})\end{array}$ \\
\hline MMP-2 & 5'-TGGCAAGTACGGCTTCTGTC-3' & 60 \\
5'-TTCTTGTCGCGGTCGTAGTC-3' & & 180 \\
& 5'-TGGTCCTGGTGCTCCTGGTG-3' & 60 \\
GAPDH & 5'-GCTGCCTGTCGGTGAGATTGG-3' & \\
& 5'-ACCACAGTCCATGCCATCAC-3' & 60 \\
\hline
\end{tabular}

A
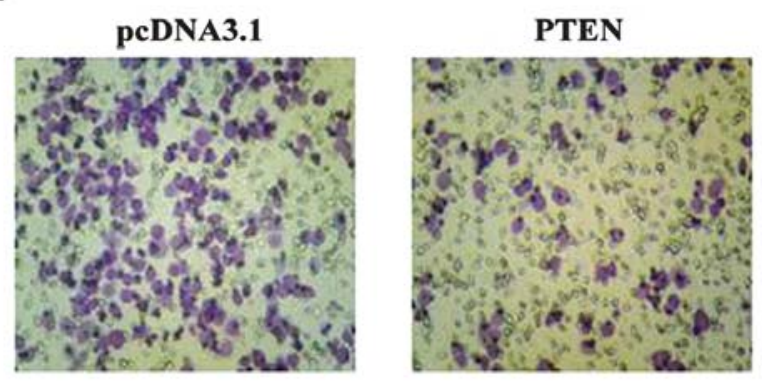

C
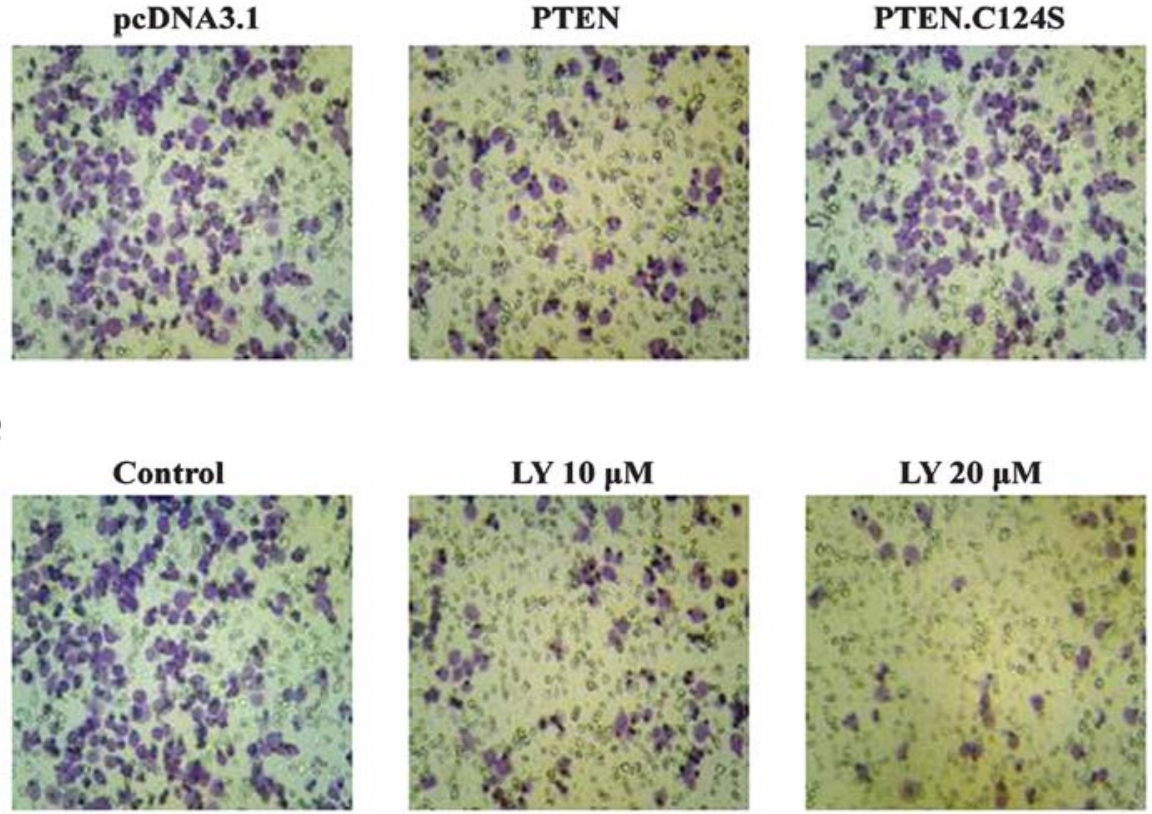

$\mathbf{E}$
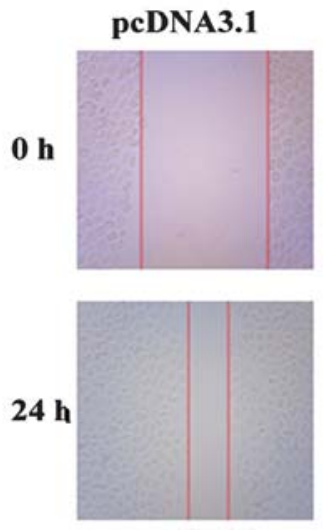

$247 \mu M$
PTEN
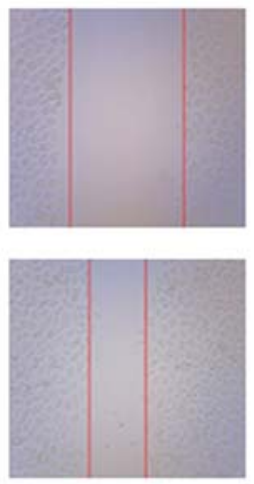

$159 \mu \mathrm{M}$
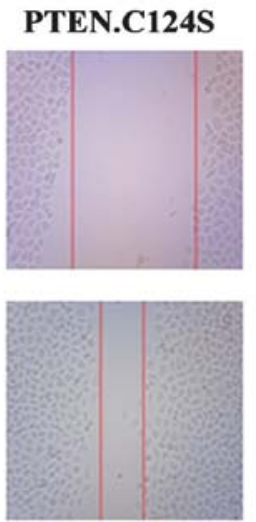

$225 \mu M$
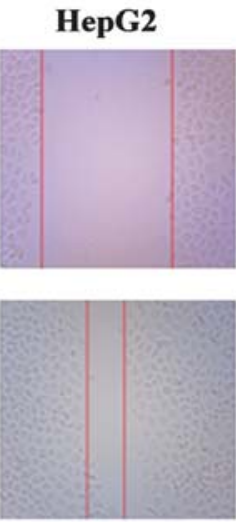

$260 \mu M$
B

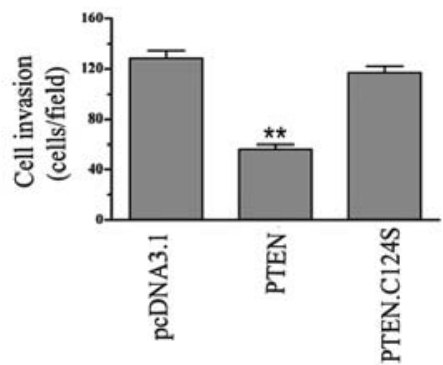

D

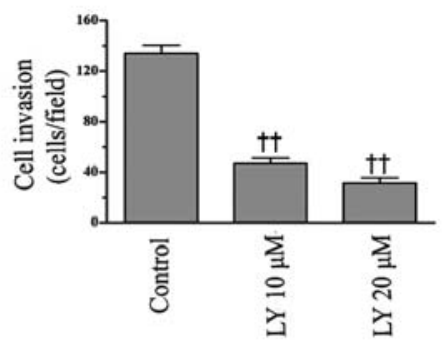

\section{LY $20 \mu M$}
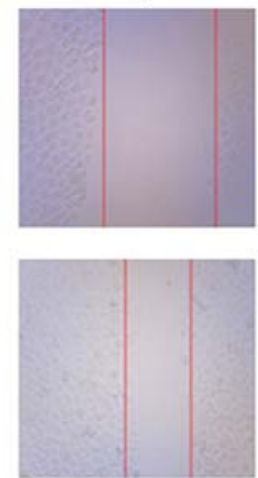

$132 \mu \mathrm{M}$
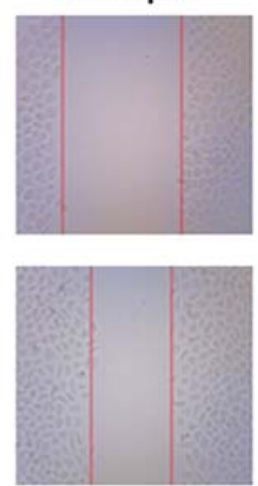

$111 \mu \mathrm{M}$

Figure 3. Overexpression of PTEN inhibits the migratory and invasive ability of HepG2 cells. The upper wells of the transwell chambers were coated with Matrigel and seeded with HepG2 cells transfected with various expression plasmids (A and B) or cells treated with LY294002 (C and D) in RPMI-1640 medium supplemented with 1\% FBS. Cell invasion was assayed after $24 \mathrm{~h}$. After removal of cells from the upper surface of the chambers, the membranes were stained with crystal violet. Invading cells were quantified by counting cells in 10 random fields at x200 magnification. Columns, mean (n=10); bars, SD; ${ }^{* *} \mathrm{P}<0.01 ;{ }^{\dagger+} \mathrm{P}<0.01$ compared to pcDNA3.1 or HepG2 controls, respectively. (E) Images of initial time point controls and $24 \mathrm{~h}$ time points show the effects of PTEN or LY294002 on the ability of cells to recover from the scratch. Red lines delineate the sides of the scratch. The numbers under each image indicate the difference between intervals at two time points. 
A

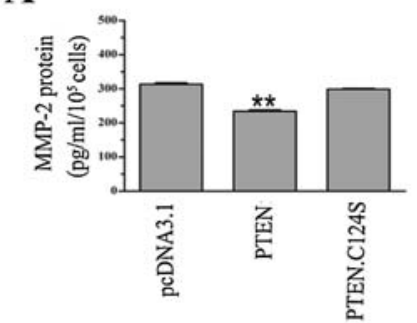

C

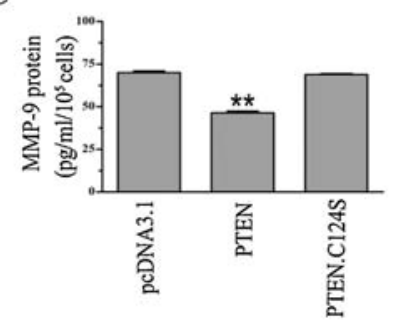

D

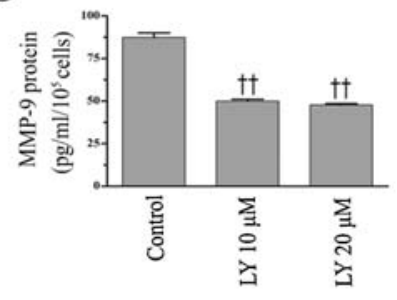

$\mathbf{E}$

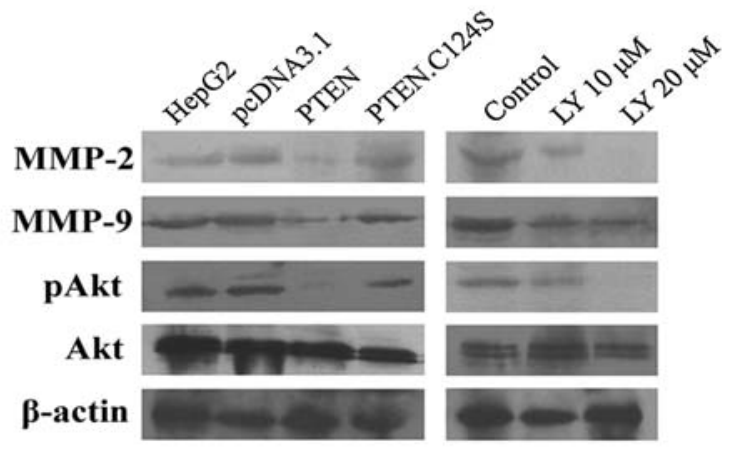

$\mathbf{F}$

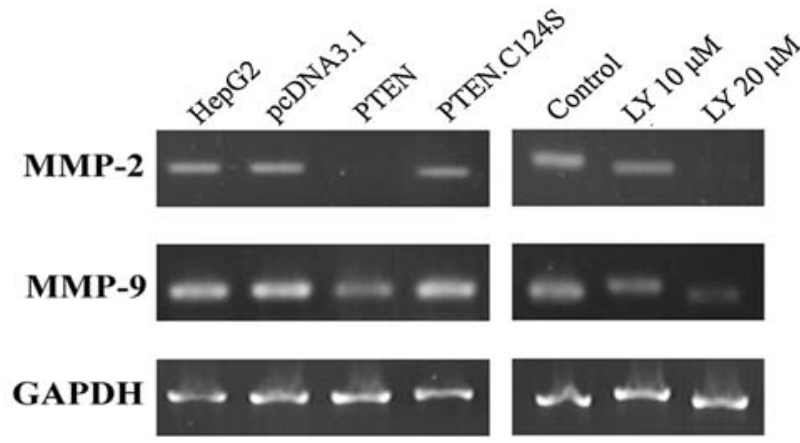

Figure 4. PTEN suppresses MMP-2 and MMP-9 expression through the PI3K/Akt pathway. MMP-2 concentrations in the various cell supernatants from HepG2 cells transfected with various expression plasmids (A) or treated with LY294002 (B) were analyzed by ELISA. Columns, mean $(\mathrm{n}=3)$; bars, $\mathrm{SD} ;{ }^{* *} \mathrm{P}<0.01 ;{ }^{\dagger} \mathrm{P}<0.01$ compared to pcDNA3.1 or HepG2 controls, respectively. MMP-9 concentrations in the various cell supernatants from HepG2 cells transfected with various expression plasmids (C) or treated with LY294002 (D) were analyzed by ELISA. Columns, mean $(\mathrm{n}=3)$; bars, $\mathrm{SD} ;{ }^{* *} \mathrm{P}<0.01 ;{ }^{\dagger} \mathrm{P}<0.01$ compared to pcDNA3.1 or HepG2 controls, respectively. (E) Expression of MMP-2 and MMP-9 in HepG2 cells transfected with various expression plasmids or treated with LY294002 was analyzed by Western blotting. B-actin was used as an internal control (F) RT-PCR analysis of MMP-2 and MMP-9 mRNA expression in HepG2 cells transfected with various expression plasmids or treated with LY294002. GAPDH was used as an internal control.

with LY294002 resulted in a significant and concentrationdependent decrease in the ability of these cells to invade through the Matrigel (Fig. 3C and D).

We also conducted a scratch assay to determine whether PTEN inhibits migration of HepG2 cells in vitro. After $24 \mathrm{~h}$, the migratory potential of PTEN-expressing HepG2 cells was significantly reduced compared to that of cells transfected with empty plasmid (Fig. 3E). In contrast, the migratory ability of PTEN.C124S-expressing HepG2 cells was not affected (Fig. 3E). We also observed that LY294002 significantly decreased the ability of $\mathrm{HepG} 2$ cells to recover from the scratch in a concentration-dependent manner (Fig. 3E). These experiments support the hypothesis that PTEN inhibits migration and invasion of $\mathrm{HepG} 2$ cells in vitro via the $\mathrm{PI} 3 \mathrm{~K} /$ Akt pathway.

PTEN downregulates MMP-2 and MMP-9 expression through the PI3K/Akt pathway. MMPs, particularly MMP-2 and MMP-9, degrade the ECM and are strongly implicated in invasion and metastasis of malignant tumor cells $(4,6)$. In light of this, we hypothesized that PTEN could inhibit HepG2 migration and invasion by suppressing the expression of MMP-2 and MMP-9. Analysis of cell supernatants by ELISA revealed that HepG2 cells transfected with empty plasmid secreted $313.52 \pm 7.38 \mathrm{pg} / \mathrm{ml} / 10^{5}$ cells of MMP- 2 and $70.05 \pm 1.75 \mathrm{pg} / \mathrm{ml} / 10^{5}$ cells of MMP-9 (Fig. 4A and C). PTEN expression in HepG2 cells significantly reduced MMP-2 levels to $234.50 \pm 6.49 \mathrm{pg} / \mathrm{ml} / 10^{5}$ cells $(\mathrm{P}<0.01$, Fig. $4 \mathrm{~A})$ and MMP-9 levels to $46.38 \pm 1.81 \mathrm{pg} / \mathrm{ml} / 10^{5}$ cells $(\mathrm{P}<0.01$, Fig. $4 \mathrm{C})$. These results were confirmed by Western blotting (Fig. 4E). To determine whether PTEN suppresses expression of MMP-2 and MMP-9 at the mRNA level, RT-PCR analysis was performed. Consistent with previous results, PTEN-expressing HepG2 cells exhibited decreased MMP-2 and MMP-9 mRNA levels (Fig. 4F). However, the expression of PTEN.C124S did not suppress the expression of MMP-2 and MMP-9 protein or mRNA (Figs. 4A and C, 4E and F).

To further define the point in the PI3K/Akt pathway at which PTEN regulates the expression of MMP-2 and MMP-9, we treated HepG2 cells with LY294002 and analyzed the expression of protein and mRNA for these two molecules. Analysis of supernatants from LY294002-treated and controltreated HepG2 cells by ELISA revealed that LY294002 significantly decreased the levels of secreted MMP-2 and MMP-9 in a concentration-dependent manner (Fig. 4B and D). These results were confirmed by Western blot and RT-PCR analysis (Fig. 4E and F). It is worth noting that decreased expression of MMP-2 and MMP-9 correlated with the inhibition of Akt phosphorylation caused by overexpression of PTEN or treatment of HepG2 cells with LY294002 (Fig. 4). These results and the results of our invasion assays indicate that PTEN inhibits invasion of $\mathrm{HepG} 2$ cells primarily by suppressing MMP-2 and MMP-9 expression. In addition, our results demonstrate that the PI3K/Akt pathway is essential for promoting the invasive behavior of HepG2 cells.

\section{Discussion}

HCC cells utilize multiple molecular signaling pathways to proliferate, invade and metastasize during the course of tumor progression. Among several independent signaling pathways, activation of the PI3K/Akt pathway is particularly important (31). Loss of PTEN, phosphorylation of Akt and activation of downstream targets coordinate complex events that initiate and sustain the malignant phenotype of tumor 
cells $(23,32)$. However, the direct effects of PTEN on the progression of HCC and the mechanisms involved in this process have not been previously reported. In this study, we provide the first evidence that PTEN not only inhibits the growth of HepG2 cells but also suppresses the invasion of these cells by inactivating the PI3K/Akt pathway.

It is known that the PI3K/Akt signaling pathway provides cells with a survival signal that allows them to proliferate and overcome apoptotic stimuli (24). Using tumor cell lines, many studies have shown that PTEN inhibits cell growth and induces cell cycle arrest and apoptosis through the PI3K/Akt pathway $(33,34)$. Our data clearly show that overexpression of PTEN in HepG2 cells inhibits their growth and induces cell cycle arrest at the G1 phase. The role of PTEN in the regulation of apoptosis is an interesting finding. In contrast to previous studies, our experiments show that PTEN does not induce apoptosis of tumor cells. Similarly, other studies have shown that exogenous PTEN suppresses cell growth and cell cycle progression without inducing apoptosis $(35,36)$. These findings possibly implicate cell type species-differences in the regulation of apoptosis by PTEN. Since the phosphatasedead PTEN mutant abolished the inhibition of cell growth and cell cycle progression of HCC cells, we conclude that PTEN exerts these effects through inhibition of the PI3K/Akt pathway.

Our study also implicates PTEN in suppressing the migration and invasion of HCC cells and provides evidence that the phosphatase activity of PTEN is required for invasion. Our conclusions are based on three major points: 1) highly invasive HepG2 cells harbor deletions in PTEN and exhibit high levels of Akt activity; 2) there was a positive correlation between Akt phosphorylation and invasion; and 3) LY294002 suppressed the invasive properties of HepG2 cells, but transfection of HepG2 cells with phosphatase-dead PTEN did not efficiently inhibit invasion. In addition, we found that transfection of HepG2 cells with wild-type PTEN, PTEN.C124S or empty plasmid or their treatment with $10 \mu \mathrm{M}$ or $20 \mu \mathrm{M}$ LY294002 did not significantly inhibit cell growth during the $24 \mathrm{~h}$ period of the migration and invasion assays (Fig. 1A and data not shown). Thus, we consider that the inhibitory effect of PTEN on the growth of HepG2 cells does not contribute to their migration and invasion.

It is currently accepted that degradation of the surrounding ECM is one of the common features of the tumor invasion process. MMPs secreted by invading tumor cells can degrade all essential components of the ECM. Therefore, MMPs have been proposed to play an important role in mediating the invasion of many cancer cell types (2-4). The invasiveness of aggressive cancer cells is in part determined by the proteolytic activity of MMPs and reflects the capacity of these enzymes to degrade the ECM (6-9). Accumulating evidence has shown that PTEN reduces the expression of MMPs and inhibits cancer cell invasion $(25,37)$. In the present study, we found that PTEN reduced MMP-2 and MMP-9 mRNA and protein levels, as determined by RT-PCR, Western blotting and ELISA. Therefore, the invasiveness of PTEN-expressing HepG2 cells was attenuated, in part, due to decreased expression of MMP-2 and MMP-9.

Given that PTEN functions to antagonize PI3K/Akt signaling and that activation of the PI3K/Akt pathway promotes the invasion of numerous tumor types, as observed in prostate cancer (25), malignant glioma (38) and esophageal cancer (39), we next sought to determine the relationship between MMP expression and Akt activation caused by phosphatase dead-PTEN expression or treatment with LY294002. As expected, the phosphatase-dead PTEN mutant did not affect Akt activation nor inhibited expression of MMPs in HepG2 cells. In contrast, there was a significant, concentration-dependent decrease in Akt phosphorylation and MMP expression upon exposure of HepG2 cells to LY294002. Previous studies support these findings and have demonstrated that treatment of cancer cells with LY294002 results in decreased expression of MMPs $(25,38)$. In addition, the data from these studies suggest that PTEN suppresses MMP-2 and MMP-9 expression at least in part by inhibiting the PI3K/Akt pathway. However, future studies are required to confirm these results in vivo.

In summary, our results demonstrate that PTEN not only inhibits the growth of HepG2 cells via cell cycle arrest, but also suppresses cell invasion in a PI3K/Akt/MMP-dependent manner. Thus, we propose that the tumor suppressor gene PTEN and its associated regulatory signaling pathways may be effective candidates for therapeutic intervention in the management of HCC.

\section{Acknowledgements}

We thank Professor Yuxin Yin (Columbia University, NY, USA) for the PTEN and PTEN.C124S plasmids.

\section{References}

1. Parkin DM, Bray F, Ferlay J and Pisani P: Global cancer statistics, 2002. CA Cancer J Clin 55: 74-108, 2005.

2. Deryugina EI and Quigley JP: Matrix metalloproteinases and tumor metastasis. Cancer Metastasis Rev 25: 9-34, 2006.

3. Fingleton B: Matrix metalloproteinases: roles in cancer and metastasis. Front Biosci 11: 479-491, 2006.

4. Egeblad M and Werb Z: New functions for the matrix metalloproteinases in cancer progression. Nat Rev Cancer 2: 161-174, 2002.

5. Nelson AR, Fingleton B, Rothenberg ML and Matrisian LM: Matrix metalloproteinases: biologic activity and clinical implications. J Clin Oncol 18: 1135-1149, 2000.

6. Björklund $\mathrm{M}$ and Koivunen E: Gelatinase-mediated migration and invasion of cancer cells. Biochim Biophys Acta 1755: 37-69, 2005.

7. Canel M, Secades P, Garzón-Arango M, et al: Involvement of focal adhesion kinase in cellular invasion of head and neck squamous cell carcinomas via regulation of MMP-2 expression. Br J Cancer 98: 1274-1284, 2008.

8. Lamar JM, Pumiglia KM and DiPersio CM: An immortalizationdependent switch in integrin function up-regulates MMP-9 to enhance tumor cell invasion. Cancer Res 68: 7371-7379, 2008.

9. Zheng H, Takahashi H, Murai Y, et al: Expressions of MMP-2, MMP-9 and VEGF are closely linked to growth, invasion, metastasis and angiogenesis of gastric carcinoma. Anticancer Res 26: 3579-3583, 2006

10. Chen JS, Wang Q, Fu XH, et al: Involvement of PI3K/PTEN/ AKT/mTOR pathway in invasion and metastasis in hepatocellular carcinoma: Association with MMP-9. Hepatol Res 39: 177-186, 2009.

11. Zhang Q, Chen X, Zhou J, et al: CD147, MMP-2, MMP-9 and MVD-CD34 are significant predictors of recurrence after liver transplantation in hepatocellular carcinoma patients. Cancer Biol Ther 5: 808-814, 2006.

12. Wu JM, Sheng H, Saxena R, et al: NF-kappaB inhibition in human hepatocellular carcinoma and its potential as adjunct to sorafenib based therapy. Cancer Lett 278: 145-155, 2009. 
13. Taras D, Blanc JF, Rullier A, et al: Pravastatin reduces lung metastasis of rat hepatocellular carcinoma via a coordinated decrease of MMP expression and activity. J Hepatol 46: 69-76, 2007.

14. Yoshida T, Hisamoto T, Akiba J, et al: Spreds, inhibitors of the Ras/ERK signal transduction, are dysregulated in human hepatocellular carcinoma and linked to the malignant phenotype of tumors. Oncogene 25: 6056-6066, 2006.

15. Yao YJ, Ping XL, Zhang H, et al: PTEN/MMAC1 mutations in hepatocellular carcinomas. Oncogene 18: 3181-3185, 1999.

16. Bae JJ, Rho JW, Lee TJ, et al: Loss of heterozygosity on chromosome 10q23 and mutation of the phosphatase and tensin homolog deleted from chromosome 10 tumor suppressor gene in Korean hepatocellular carcinoma patients. Oncol Rep 18: 1007-1013, 2007.

17. Horie Y, Suzuki A, Kataoka E, et al: Hepatocyte-specific PTEN deficiency results in steatohepatitis and hepatocellular carcinomas. J Clin Invest 113: 1774-1783, 2004.

18. Hu TH, Huang CC, Lin PR, et al: Expression and prognostic role of tumor suppressor gene PTEN/MMAC1/TEP1 in hepatocellular carcinoma. Cancer 97: 1929-1940, 2003.

19. Vinciguerra M and Foti M: PTEN at the crossroad of metabolic diseases and cancer in the liver. Ann Hepatol 7: 192-199, 2008.

20. Li L and Ross AH: Why is PTEN an important tumor suppressor? J Cell Biochem 102: 1368-1374, 2007

21. Rossi DJ and Weissman IL: PTEN, tumorigenesis, and stem cell self-renewal. Cell 125: 229-231, 2006.

22. Wu Z, McRoberts KS and Theodorescu D: The role of PTEN in prostate cancer cell tropism to the bone micro-environment. Carcinogenesis 28: 1393-1400, 2007.

23. Chalhoub $\mathrm{N}$ and Baker SJ: PTEN and the PI3-kinase pathway in cancer. Annu Rev Pathol 4: 127-150, 2009.

24. Downward J: PI 3-kinase, Akt and cell survival. Semin Cell Dev Biol 15: 177-182, 2004.

25. Shukla S, Maclennan GT, Hartman DJ, Fu P, Resnick MI and Gupta S: Activation of PI3K-Akt signaling pathway promotes prostate cancer cell invasion. Int J Cancer 121: 1424-1432, 2007.

26. Fu Y, Zhang Q, Kang C, et al: Inhibitory effects of adenovirus mediated COX-2, Akt1 and PIK3R1 shRNA on the growth of malignant tumor cells in vitro and in vivo. Int J Oncol 35: 583-591, 2009

27. Diaz R, Nguewa PA, Diaz-Gonzalez JA, et al: The novel Akt inhibitor Palomid 529 (P529) enhances the effect of radiotherapy in prostate cancer. Br J Cancer 100: 932-940, 2009.
28. Shen WH, Balajee AS, Wang J, et al: Essential role for nuclear PTEN in maintaining chromosomal integrity. Cell 128: 157-170, 2007.

29. Kang-Park S, Lee YI and Lee YI: PTEN modulates insulin-like growth factor II (IGF-II)-mediated signaling; the protein phosphatase activity of PTEN downregulates IGF-II expression in hepatoma cells. FEBS Lett 545: 203-208, 2003.

30. Meng F, Henson R, Wehbe-Janek H, Ghoshal K, Jacob ST and Patel T: MicroRNA-21 regulates expression of the PTEN tumor suppressor gene in human hepatocellular cancer. Gastroenterology 133: 647-658, 2007.

31. Cully M, You H, Levine AJ and Mak TW: Beyond PTEN mutations: the PI3K pathway as an integrator of multiple inputs during tumorigenesis. Nat Rev Cancer 6: 184-192, 2006.

32. Salmena L, Carracedo A and Pandolfi PP: Tenets of PTEN Tumor Suppression. Cell 133: 403-414, 2008.

33. Gan YH and Zhang S: PTEN/AKT pathway involved in histone deacetylases inhibitor induced cell growth inhibition and apoptosis of oral squamous cell carcinoma cells. Oral Oncol 45: E150-E154, 2009.

34. Li X, Lin G, Wu B, Zhou X and Zhou K: Overexpression of PTEN induces cell growth arrest and apoptosis in human breast cancer ZR-75-1 cells. Acta Biochim Biophys Sin 39: 745-750, 2007.

35. Moon SK, Kim HM and Kim CH: PTEN induces G1 cell cycle arrest and inhibits MMP-9 expression via the regulation of NFkappaB and AP-1 in vascular smooth muscle cells. Arch Biochem Biophys 421: 267-276, 2004.

36. Minaguchi T, Mori T, Kanamori Y, et al: Growth suppression of human ovarian cancer cells by adenovirus-mediated transfer of the PTEN gene. Cancer Res 59: 6063-6067, 1999.

37. Furukawa K, Kumon Y, Harada H, et al: PTEN gene transfer suppresses the invasive potential of human malignant gliomas by regulating cell invasion-related molecules. Int J Oncol 29: 73-81, 2006.

38. Kubiatowski T, Jang T, Lachyankar MB, et al: Association of increased phosphatidylinositol 3-kinase signaling with increased invasiveness and gelatinase activity in malignant gliomas. J Neurosurg 95: 480-488, 2001.

39. Li B, Tsao SW, Li YY, et al: Id-1 promotes tumorigenicity and metastasis of human esophageal cancer cells through activation of PI3K/AKT signaling pathway. Int J Cancer 125: 2576-2585, 2009. 